\title{
Suppression of MafA mRNA with siRNA prevents adipose cell differentiation in 3T3-L1 cells
}

\author{
MARIKO TSUCHIYA ${ }^{1}$, ATSUSHI MAEDA ${ }^{1}$, AYUME SUZUKI $^{1}$, KAZUKI YASUDA ${ }^{2}$, \\ TAKUMI YOSHIDA ${ }^{3}$, KOSAKU NITTA ${ }^{3}$ and KEN TSUCHIYA ${ }^{3}$ \\ ${ }^{1}$ Institute of Geriatrics, Tokyo Women's Medical University, 2-15-1 Shibuya, Shibuya-ku, Tokyo 150-0002; \\ ${ }^{2}$ International Medical Center of Japan, 1-21-1 Toyama, Shinjuku-ku, Tokyo 162-8655; \\ ${ }^{3}$ Department of Medicine IV, Tokyo Women's Medical University, \\ 8-1 Kawada-cho, Shinjuku-ku, Tokyo 162-8666, Japan
}

Received December 29, 2008; Accepted March 26, 2009

DOI: 10.3892/ijmm_00000186

\begin{abstract}
One of the large Mafs, MafA protein, is a strong transactivator of insulin in pancreatic $B$ cells. Mafs are also known to play important roles in a variety of developmental and differentiation processes in many organs and tissues. Adipocytes are highly involved in insulin actions and glucose and lipid metabolism, and their proliferation and differentiation is regulated by coordination of several signal transduction and transcriptional factors, including members of the Maf family. To explore the role of MafA in adipocytes, we modified the MafA mRNA level in cultured adipocytes by the RNA interference technique and analyzed the resulting morphological changes and changes in expression of related genes. MafA siRNA was transfected into 3T3-L1 adipocytes. Expression of MafA was confirmed by real-time PCR and Western blotting. Expression of adipocytokines and transcriptional factors was also measured by real-time PCR. Cells were examined for morphological changes and lipid accumulation by microscopy. The MafA expression level in the MafA-siRNA-transfected pre-adipocytes was reduced by $\sim 30 \%$ on day 0 pre-induction and by $\sim 70 \%$ on day 3 post-induction, in comparison with stop-siRNA-transfected cells. Cell growth and lipid droplet accumulation were prevented by MafA mRNA suppression, and peroxisome proliferator-activated receptor (PPAR) $\gamma 2$ and CCAAT/enhancerbinding proteins $(\mathrm{C} / \mathrm{EBP}) \alpha$, both of which are transcriptional factors essential for adipocyte differentiation, were downregulated. Expression of the genes encoding the adipocytokines, adiponectin and adipsin was also suppressed. The results suggested a possible role of the transcriptional factor MafA in regulation of adipocyte function and differentiation.
\end{abstract}

Correspondence to: Dr Ken Tsuchiya, Department of Medicine IV, Tokyo Women's Medical University, 8-1 Kawada-cho, Shinjuku-ku, Tokyo 162-8666, Japan

E-mail: tsuchiya@kc.twmu.ac.jp

Key words: adipocyte, mafA, siRNA, transcriptional factor

\section{Introduction}

The large Maf proteins are a family of transcription factors characterized by a typical bZip structure, which is a motif for protein dimerization and DNA binding, and they were reported to regulate several distinct developmental processes, cell differentiation, and the establishment of cell functions (1-3). One of the large Mafs, MafA protein, is a strong transactivator of insulin in pancreatic $\beta$ cells $(4,5)$, and there are many reports that MafA transcription factor contributes to $\beta$ cell function and differentiation (6-9). One study demonstrated that loss of MafA protein causes a decrease in insulin gene expression in glucotoxic $B$ cells (10), and another study showed a marked reduction of insulin transcription in MafA-deficient mice, even though the insulin content of $\beta$ cells was not significantly reduced (11).

On the other hand, it is well known that the Mafs play important roles in a variety of developmental and differentiation processes in many organs, tissues, and cells, including the pancreas (12), lens (13), myeloma cells (14), and cartilage (15). Adipocytes are closely related to insulin action and glucose as well as lipid metabolism, and are candidates for Maf-related cells (16). Their differentiation and proliferation is regulated by coordination of several signal transduction and transcriptional factors. Moreover, in a previous study, we elucidated the expression profiles of related genes in the pancreas of mafA-siRNA-treated mice and observed changes in MafA mRNA expression that were likely linked to the level of expression of adipocytokines (17), suggesting several potential biological roles of MafA in the regulation of adipose tissue differentiation and development. The purpose of the present in vitro study was to determine whether MafA is expressed during adipocyte differentiation and the effect of its expression on the gene profile related to adipogenesis and adipocytokines, by using the RNA interference technique.

\section{Materials and methods}

Cell culture. Mouse 3T3-L1 cells from American Type Culture Collection (Manassas, VA) were maintained in high-glucose Dulbecco's modified Eagle medium (DMEM) (Invitrogen, 
Carlsbad, CA) containing $10 \%$ calf serum. At 2 days postconfluence, 3T3-L1 pre-adipocytes were induced to differentiate (day 0) by addition of DMEM supplemented with $10 \%$ fetal bovine serum (FBS) containing $1 \mu \mathrm{g} / \mathrm{ml}$ insulin, $0.25 \mu \mathrm{M}$ dexamethasone (Sigma, St. Louis, MO), and 0.5 mM 3-isobutyl-1methylxanthine (IBMX) (Sigma). After 2 days, culture medium was replaced with medium supplementated with $10 \%$ FBS and $1 \mu \mathrm{g} / \mathrm{ml}$ insulin, and 2 days later the medium was replaced with DMEM supplemented with $10 \%$ FBS. The medium was changed every 2 days thereafter until cells were differentiated.

MafA siRNA. A designed MafA small interfering RNA (siRNA) oligomer constructed in a plasmid was purchased from Takara Bio (Takara Bio Co., Ohtsu, Shiga). The target and designed siRNA sequences are shown in Table I.

SiRNA transfection of cultured cells and the experimental protocol. 3T3-L1 cells were transfected by using TransIT-LT1 transfection reagent according to the manufacturer's instructions (Mirus, Madison, WI). Briefly, a $5 \times 10^{5}$ cell sample was transfected with $15 \mu \mathrm{g}$ of MafA siRNA dissolved in $1.5 \mathrm{ml}$ of serum-free DMEM culture medium to which $45 \mu 1$ of TransIT-LT1 was added. Cells transfected with stop siRNA were used as a control. The culture medium was replaced with regular culture medium $48 \mathrm{~h}$ later. After an additional $48 \mathrm{~h}$, cells were selected with $14 \mu \mathrm{g} / \mathrm{ml}$ puromycin and maintained in puromycin selection medium for more than 2 weeks. The morphological appearance of cultured preadipocytes before induction and 3 and 7 days after induction were compared. Cells were harvested 3 and 7 days after induction, and mRNA and protein were extracted. Downregulation of MafA was confirmed by estimating the mRNA level by real-time PCR and estimating protein expression by Western blotting.

Reverse-transcription $(R T)$ and real-time PCR. RNA isolation and real-time PCR were performed as previously described (18). Briefly, total RNA was isolated from cells with TRIzol Reagent (Invitrogen, Carlsbad, CA). Relative quantitation the real-time PCR method was performed by using SYBR Green PCR Reagents and an ABI PRISM 7500 Sequence Detection System (PE Applied Biosystems, Foster City, CA) according to the manufacturer's instructions. Reactions were performed using $1.0 \mu \mathrm{l}$ of RNA at a concentration of $50 \mathrm{ng} / \mu 1 \mathrm{in} \mathrm{a}$ reaction volume of $25 \mu \mathrm{l}$. RT was performed at $37^{\circ} \mathrm{C}$ for $120 \mathrm{~min}$, followed by PCR consisting of AmpliTaq activation for $10 \mathrm{~min}$ at $95^{\circ} \mathrm{C}$, then 40 cycles of heating to $95^{\circ} \mathrm{C}$ for 15 sec and cooling to $60^{\circ} \mathrm{C}$ for $1 \mathrm{~min}$, and the mRNA levels were normalized to the levels of GAPDH mRNA. Changes in gene expression level of each in the protocol were investigated by real-time PCR with specific primer sets. The primer sets for genes related to adipocyte differentiation, adipocytokines, etc., were synthesized as shown in Table I, and we used GAPDH (Applied Biosystems, P/N 4308313) as the internal standards. The real-time PCR was performed by the same protocol as described for this method.

Histochemical analysis of culture cells. The morphology of 3T3-L1 cells was directly observed under a microscope and lipid droplets were stained with oil-red. 3T3-L1 cells were
Table I. Target sequence and designed siRNA sequence.

MafA NM 194350

Target sequence

AGCGGGACCCTGTACAAGGA

Sense oligo

gtttAGTGGGACTTGTACAGG

GAACGTGTGCTGTCCGTTCC TTGTACAGGTCCCGCTTTTTT

Antisense oligo atgcAAAAAAGCGGGACCTGTA CCAGGAACGGACAGCACACG TTCCCTGTACAAGTCCCACT

Vector

pcPURmU6icassette

pcPURmU6biSTOP

Sense Oligo

GTTTTTTTTTT

Antisense Oligo

ATGCAAAAAAA

Primers for real-time PCR

\begin{tabular}{lcc}
\hline Gene & Forward & Reverse \\
\hline mafA & ccagctggtatccatgtcc & ttctgtttcagtcggatgacc \\
mafB $(44)$ & aacgctgcaactctcaagtg & gggacttgaacaccaccatt \\
$P P A R \gamma 2$ & tgcagctcaagctgaatcac & cacgtgctctgtgacgatct \\
C/EBP & catgccgggagaactctaac & ctctggaggtgactgctcatc \\
Adipsin & tcaatcatgaaccggacaac & aatggtgactaccccgtcat \\
Acrp30 & ccaatgtacccattcgcttt & tgctgccgtcataatgattc \\
(Adiponectin) & & \\
\hline
\end{tabular}

washed twice with PBS and fixed with $10 \%$ formalin in PBS for $15 \mathrm{~min}$. After two washes in PBS, cells were stained with $60 \%$ filtered Oil-Red-O stock solution (150 mg Oil-Red-O in $50 \mathrm{ml}$ isopropanol) for $20 \mathrm{~min}$, washed with $60 \%$ isopropanol, and maintained under PBS.

Western blotting. Cells were immersed in a lysis buffer $(20 \mathrm{mM}$ Tris buffer, pH 7.5, containing $1 \mathrm{mM}$ phenylmethylsulfonyl fluoride, $10 \mu \mathrm{g} / \mathrm{ml}$ aprotinin from bovine lung (Wako, Tokyo), $2 \mathrm{mM}$ DL-dithiothreitol, $1 \%$ polyoxyethylene sorbitan monolaurate, and $1 \mathrm{mM}$ ethylenediamine tetraacetate), and were homogenized on ice. After centrifugation at 5,000 rpm for $10 \mathrm{~min}, 30 \mu \mathrm{g}$ of protein from each was suspended in a loading buffer, separated on a $12.5 \%$ polyacrylamide gel (Readygels J, Bio-Rad, Tokyo), and electrophoretically transferred to a nitrocellulose membrane. Membranes were blocked with 3\% skim milk for $1 \mathrm{~h}$ at room temperature. A primary antibody against MafA protein (BL1069, Bethyl Laboratories Inc., Montgomery, TX) and a primary antibody against B-actin (A1978, Sigma Inc., St. Louis, MO) were used at a 1:3,000 dilution and applied overnight at $4^{\circ} \mathrm{C}$. After two 10-min washing steps with washing buffer $(0.3 \%$ Tween-20 in PBS), the membrane was incubated with horseradish-peroxidase-conjugated immunoglobulins (Dako, Glostrup, Denmark) for $1 \mathrm{~h}$ at room temperature, then with an ECL Advance Western Blotting Detection Kit (GE Healthcare, Bukinghamshire, UK), and, finally, x-ray film was exposed to the membrane. 
a)

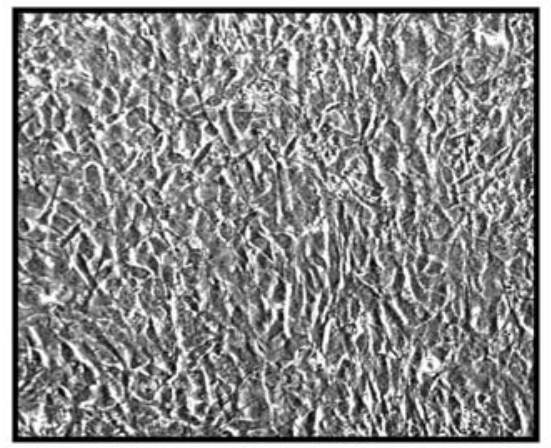

Before induction

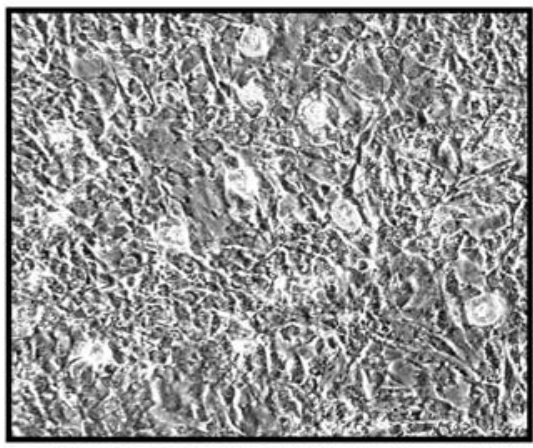

post-induction

b)

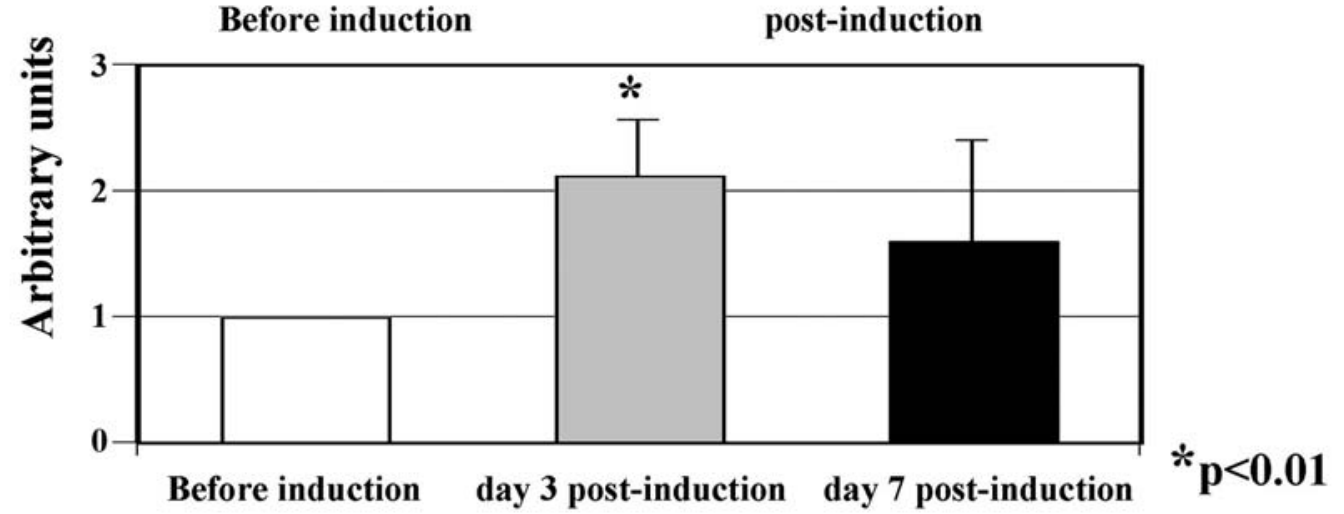

c)

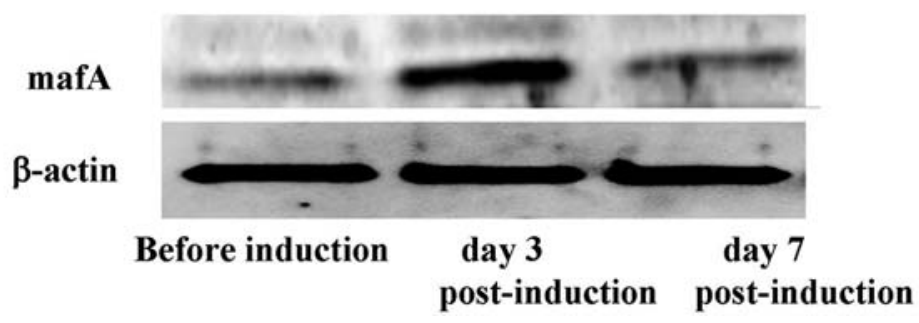

Figure 1. Histological appearance, mafA mRNA and protein expression during differentiation of 3T3-L1 cells. (a) Differentiation of 3T3-L1 cells in pre- and post-induction. (b) Real-time PCR demonstrated that mafA mRNA increased during differentiation on day 3 post-induction and slightly decreased on day 7 post-induction. The bars represent mean values $\pm \mathrm{SE}$. ${ }^{*} \mathrm{P}<0.01$. (c) Representative results of Western blotting. Protein expression of mafA increased in tandem with mRNA expression during differentiation.

Statistical analysis. All results are expressed as means \pm SEM. Differences in expression levels were analyzed by the paired Student's t test with Bonferroni correction, and differences were considered significant when the p-value was $<0.05$. The experiments were repeated 5 times. All data are reported as means \pm SE. Differences between groups were analyzed by Student's t test and two-way ANOVA by using the software program 'Stat View', and p-values $<0.05$ were considered significant.

\section{Results}

Expression of MafA in 3T3-L1 cells. The typical histological changes in 3T3-L1 cells during differentiation are shown in Fig. 1a. Cell volume increased after differentiation, indicating the presence of lipid droplets, and MafA mRNA expression was confirmed by real-time PCR. MafA mRNA expression was measured in 3T3-L1 cells, and as shown in Fig. 1b, the expression level increased on day 3 post-induction, and then decreased on day 7 post-induction. Protein expression of mafA is shown in Fig. 1c, and it increased in tandem with mRNA expression during differentiation.

Suppression of MafA mRNA in 3T3-L1 cells by siRNA. The relative expression level of mRNA was determined by the real-time PCR method to compare the MafA-siRNA-treated and stop-siRNA-treated cells. An $\sim 30 \%$ reduction in MafA mRNA expression in the 3T3-L1 pre-adipocytes was achieved in vitro by siRNA transfection. The MafA mRNA expression in the stop-siRNA-treated cells increased on day 3 post-induction, and then tended to decrease on day 7 postinduction, same as in non-treated cells (Fig. 1b). By contrast, the change in MafA expression did not occur and no transient increase in mRNA on day 3 was observed in the MafAsiRNA-treated cells. MafA mRNA expression was $\sim 70 \%$ reduced in the MafA-siRNA-treated cells in comparison with the stop-siRNA-treated cells on day 3 post-induction. The suppressive effect of siRNA on MafA expression persisted for 7 days. The time course of mRNA expression in the stopsiRNA-treated and MafA-siRNA-treated cells is compared in 
a)

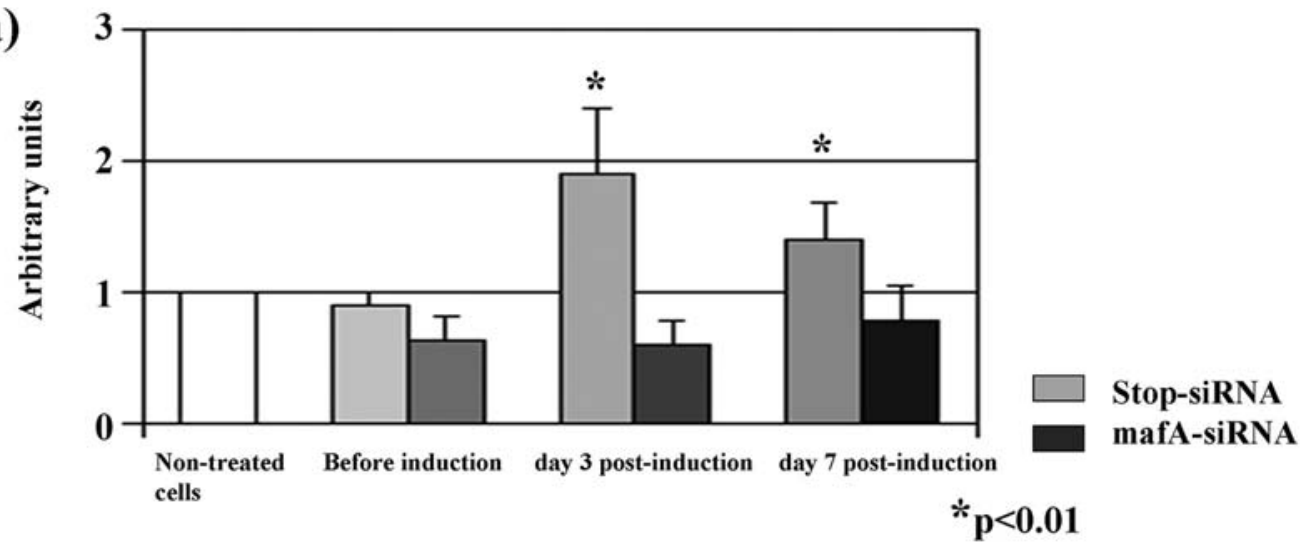

b)

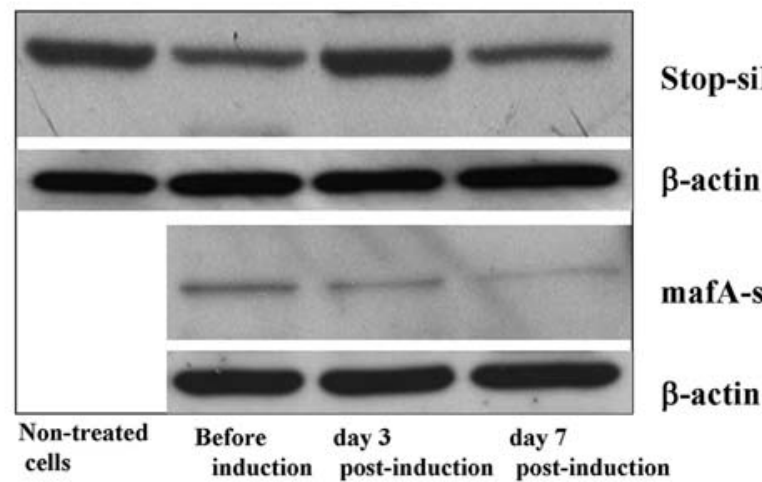

Figure 2. MafA mRNA and protein expression in siRNA-treated 3T3-L1 cells. (a) Real-time PCR mafA mRNA expression was suppressed during differentiation of mafA-siRNA-treated cells in comparison with stop-siRNA treated cells. (b) Representative results of Western blotting. Protein expression increased during differentiation in the stop-siRNA-treated control cells, but the increase was reduced in the mafA-siRNA-treated cells. The bars represent mean values \pm SE. ${ }^{*} \mathrm{P}<0.01$.

Fig. 2a. Western blot analysis was performed to confirm the results of real-time PCR. Fig. $2 b$ shows a representative blot and data demonstrating protein expression of MafA, and that increased post-induction in the stop-siRNA-treated cells. By contrast, MafA expression was significantly down-regulated in the MafA-siRNA-treated cells.

Histological changes in 3T3-L1 cells after siRNA treatment. The effects of suppression of MafA mRNA expression on cell morphology are shown in Fig. 3. The morphology of the cells was different from that of the stop-siRNA-treated cells after differentiation (Fig. 3a). The cytoplasm of the spindle-shape cells observed in the pre-adipocytes expanded during the differentiation of stop-siRNA-treated cells, whereas the shape of the MafA-siRNA-treated cells remained the same. Oil-Red-O staining demonstrated that no lipid droplets accumulated in the MafA-suppressed adipocytes (Fig. 3b). On the other hand, there were no major differences between the stop-siRNAtreated cells and the non-treated normal cells when examined microscopically and after Oil-Red-O staining.

Changes in the mRNA expression level of related genes. Adipose differentiation is regulated by sequential activation of several transcriptional factors, including peroxisome proliferator-activated receptor (PPAR) $\gamma 2$ and CCAAT/ enhancer-binding proteins $(\mathrm{C} / \mathrm{EBP}) \alpha$. Both factors are essential for adipocyte differentiation. Modulation of MafA
mRNA expression affects the expression level of other transcriptional factors, since histological examination showed that no lipid droplets accumulated. Both PPAR $\gamma 2$ and $\mathrm{C} / \mathrm{EBP} \alpha$ are expressed in pre-adipocytes, and their expression level increased during maturation (Fig. 4). The change in expression level of $\mathrm{C} / \mathrm{EBP} \alpha$ was more prominent than the change in PPAR $\gamma 2$ during differentiation. MafA mRNA suppression by siRNA almost completely abolished the expression of PPAR $\gamma 2$, but $\mathrm{C} / \mathrm{EBP} \alpha$ expression persisted throughout differentiation, although the level of expression was lower. MafA siRNA affected the level of expression of the two transcriptional factors in a different manner. The changes in expression of the adipocytokines adiponectin and adipsin are shown in Fig. 5. mRNA expression of both genes was suppressed by siRNA. The results for the adipocytokine mRNA level are consistent with the results of Oil-Red-O staining of the adipocytes in which lipid droplet accumulation did not occur.

\section{Discussion}

In our previous study we showed that suppression of MafA mRNA expression in the pancreas in vivo induced downregulation of gene expression of adipocytokines (adipsin and adiponectin) as well as pancreatic hormones (17). Although expression of adipocytokine genes has not been reported in the pancreas, adiponectin receptor is expressed in pancreatic islets. The expression of the adiponectin gene detected in the form of 
a)

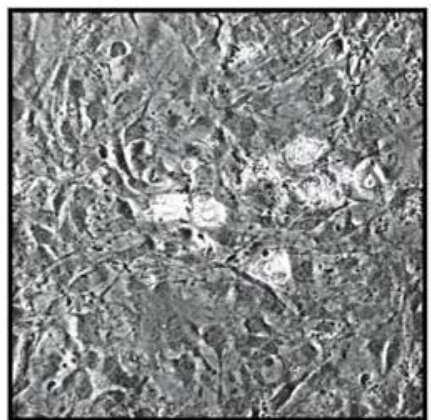

b)

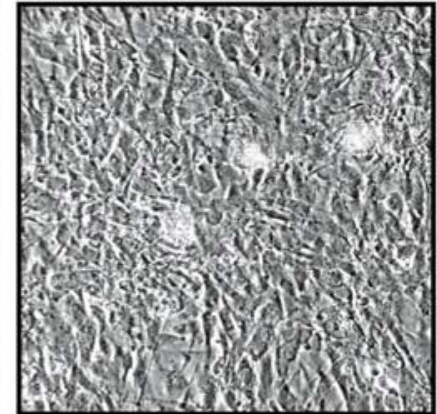

day 7 post-induction (stop-siRNA-treated cells)

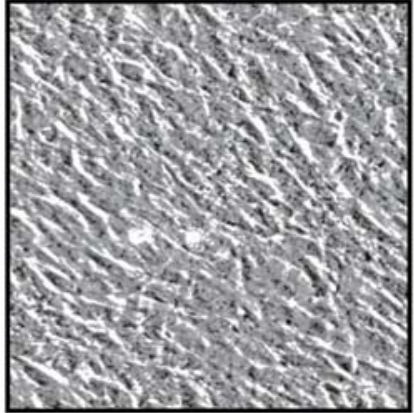

day 7 post-induction

(maf-siRNA-treated cells)

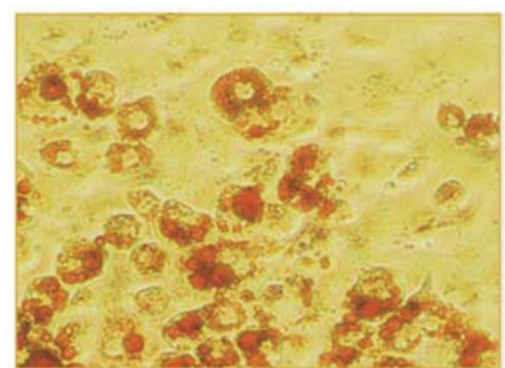

day 7 post-induction (non-treated cells)

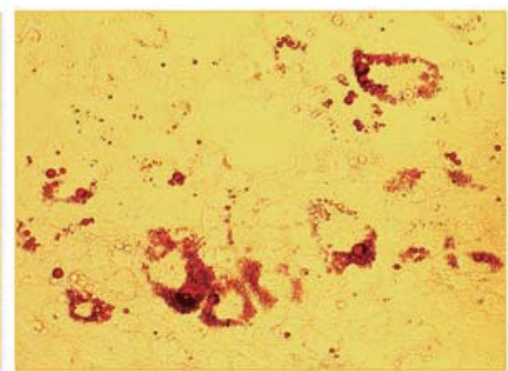

day 7 post-induction (stop-siRNA-treated cells)

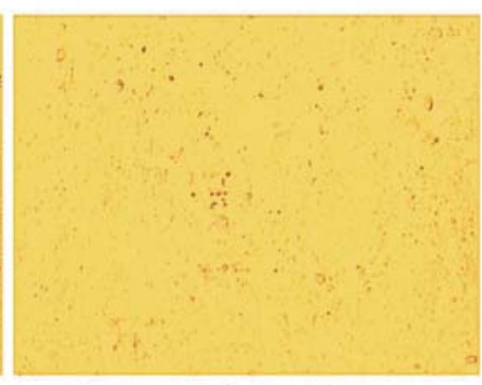

day 7 post-induction (stop-siRNA-treated cells)

Figure 3. Comparison between histological changes and the Oil-Red-O staining of non-treated, stop-mafA-siRNA- and mafA-siRNA-treated cells. (a) Histological appearance, (b) Oil-Red-O staining. No intracellular lipid staining was observed in the mafA-siRNA-treated cells.

a) PPAR $\gamma 2$

b) $\mathrm{C} / \mathrm{EBR} \alpha$
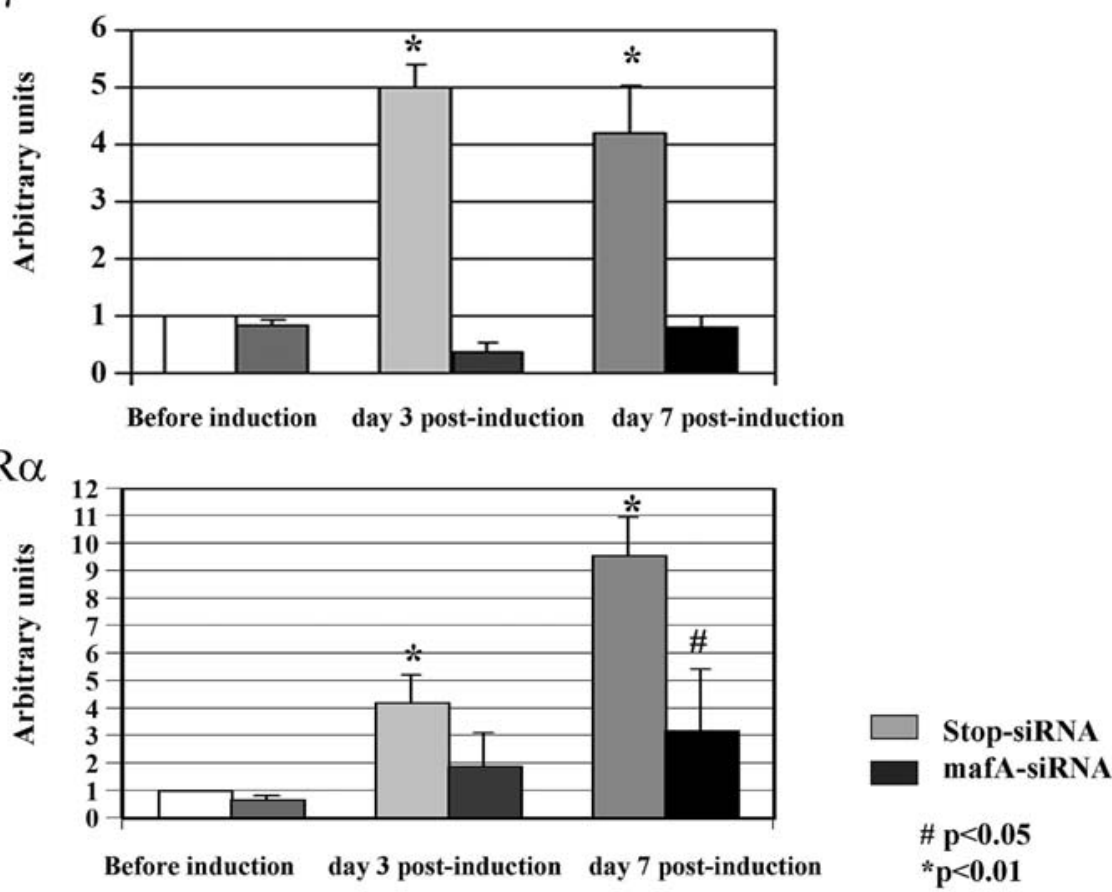

Figure 4. Real-time PCR of genes in the mafA-siRNA-treated cells, showing significant decreases in transcriptional mRNA. (a) PPAR $\gamma$ mRNA, (b) C/EBP $\alpha$ mRNA, compared with the stop-siRNA-treated cells. The bars represent mean values $\pm \mathrm{SE}$. ${ }^{\sharp} \mathrm{P}<0.05,{ }^{*} \mathrm{P}<0.01$.

mRNA in the pancreas occurred in intra-pancreatic adipose tissue or other cells, and thus MafA is involved in adipocyte differentiation and regulation of lipid metabolism through adipokine regulation in the pancreas in addition to its effects on $B$ cells (4-6). We confirmed the expression of mafA in adipocytes, and we investigated the expression of mafA in mouse pancreas and adipose tissue. We performed qRT-PCR with the same primers used in the adipocytes. We compared 
a) adiponectin

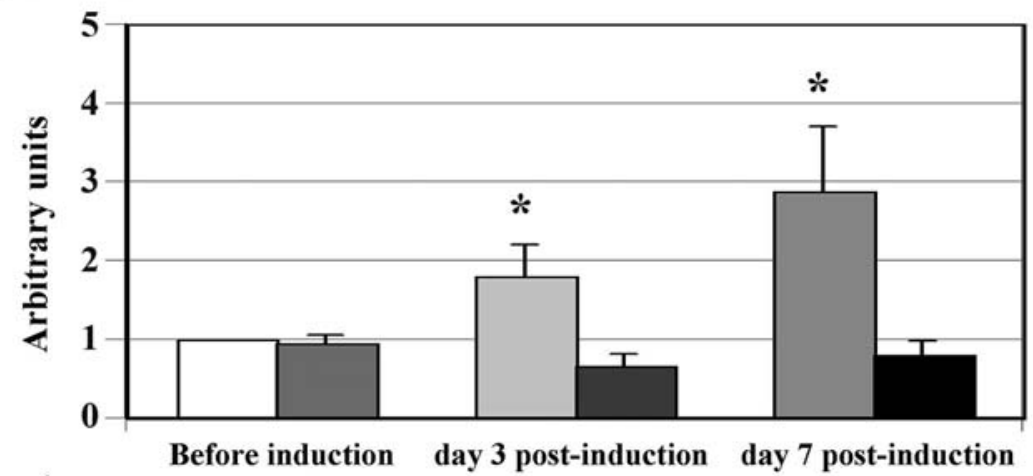

b) adipsin

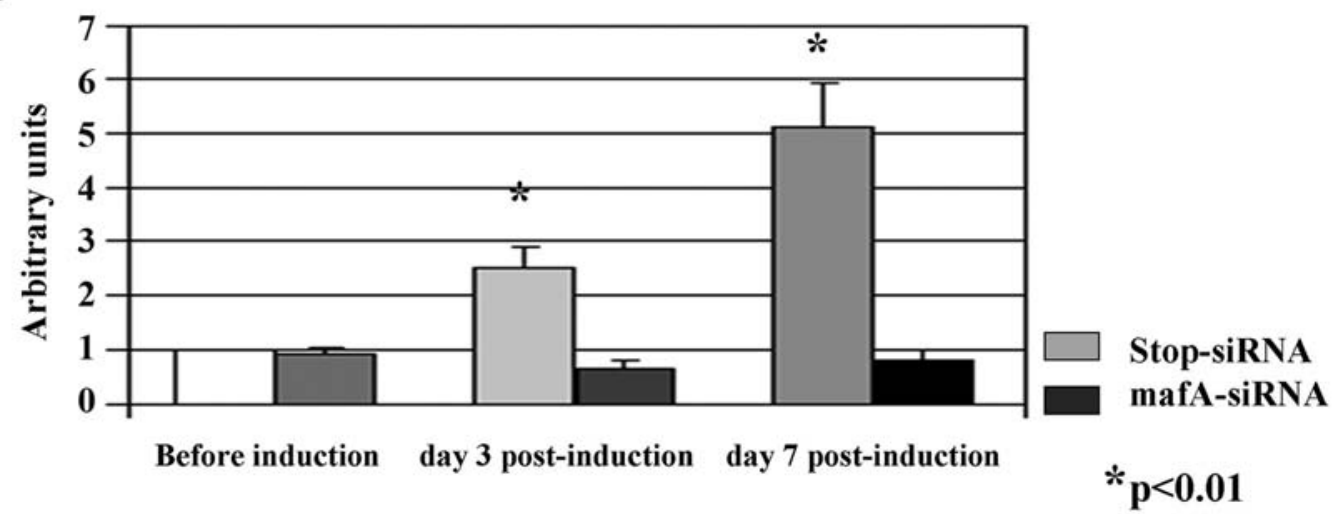

Figure 5. Real-time PCR of genes in the mafA-siRNA-treated cells, showing significant decreases in adipocytokine mRNA. (a) Adiponectin mRNA, (b) adipsin mRNA, compared with the stop-siRNA-treated cells. The bars represent mean values $\pm \mathrm{SE}$. ${ }^{*} \mathrm{P}<0.01$.

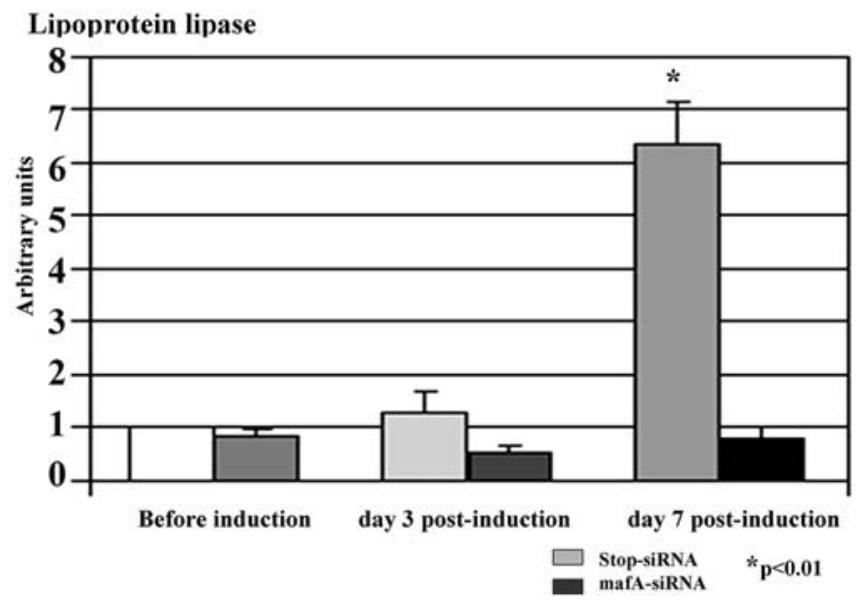

Figure 6. Results of real-time PCR of genes in the mafA-siRNA-treated cells, showing significant decreases in lipoprotein lipase mRNA Comparison between lipoprotein lipase mRNA in the mafA-siRNA-treated cells and in the stop-siRNA-treated cells. The bars represent mean values \pm SE. ${ }^{*} \mathrm{P}<0.01$.

the level of mafA mRNA expression in mouse pancreas and adipose tissue, and 3T3-L1 cells before induction, and the ratio of the expression level was 1:1.92:7.8. Then, to explore the role of MafA in adipose tissue in the present study we investigated alterations in the expression of MafA-related genes in a cultured adipocyte cell line, 3T3-L1 cells, after MafA mRNA interference.

The MafA mRNA expression level in mafA-siRNA-treated pre-adipocytes was reduced by $\sim 30 \%$ in comparison with the stop-siRNA-treated pre-adipocytes on day 0 , before induction. Although the degree of the suppressive effect on mRNA expression was not high, the increased mRNA level observed on day 3 post-induction in the stop-siRNA-treated cells was completely absent in the siRNA-treated cells. MafA mRNA expression was $\sim 70 \%$ reduced on day 3 in the MafA-siRNAtreated cells in comparison with the stop-siRNA-treated cells since MafA mRNA induction as observed in non-treated 3T3-L1 cells was suppressed. We therefore think that suppression of the mRNA increase in the stop-siRNA-treated cells after induction affected other genes related to adipocyte differentiation. In addition, we used an siRNA-expressing plasmid DNA (pDNA) instead of the oligonucleotide form of siRNA to achieve longer mRNA suppression $(21,22)$. The suppression of MafA mRNA continued for at least 7 days during differentiation.

MafA mRNA suppression induced morphological changes in 3T3-L1 cells during differentiation. Typical histological changes in 3T3-L1 cells are shown in Fig. 1a, which shows that the cytoplasm of the spindle-shape cells expanded after differentiation. This morphological change was not observed during MafA siRNA suppression, and, as shown in Fig. 3, no expansion of the cytoplasm was observed. Since lipid droplets form in adipocytes, Oil-Red-O staining revealed red droplets 
in the cytoplasm of fully differentiated adipocytes (Fig. 3), but the formation of lipid droplets was decreased when induction of MafA mRNA was suppressed. Since lipid droplet formation is essential to the adipocyte differentiation, MafA plays a critical role in the process of adipocyte differentiation.

Several genes were recognized for the differentiation and function of a 3T3-L1 cell, including the genes encoding the peroxisome proliferator-activated receptor PPAR $\gamma 2$ and $\mathrm{CCAAT}$ /enhancer-binding proteins $\mathrm{C} / \mathrm{EBP} \alpha$ and the level of expression of both genes increased during adipocyte differentiation (19). PPAR $\gamma 2$ plays a leading role in the synthesis and accumulation of lipid droplets in adipocytes, and $\mathrm{C} / \mathrm{EBP} \alpha$ is critical to the establishment of insulin sensitivity. In addition to these transcriptional factors, Serria et al reported that expression of c-Maf, another member of the maf family, is down-regulated during 3T3-L1 cell differentiation and proliferation (20). At the molecular level, the mRNA expression levels of the PPAR $\gamma$ gene or C/EBP gene, which encode the master adipogenic transcriptional factors, are markedly suppressed by mafA-siRNA treatment, i.e., by suppression of mafA expression. Since the primary purpose of this paper was to describe cultured 3T3-L1 cells expressing the mafA gene and how modulation of the mafA expression level affected histological changes and the gene expression profile, we are now focusing on the role of transcriptional factor mafA in 3T3-L1 cells. Our speculation concerning the direct effect of mafA on these transcriptional factors is based on the data obtained by quantitative real-time PCR. Thus, it is likely that the mafA gene plays a role in the differentiation of 3T3-L1 adipocytes. However, there are no direct observations of the effects of mafA expression on these transcriptional factors. Thus, additional experiments with supporting data are needed to clarify the precise biological properties of mafA and the mechanism of its role in adipocyte differentiation.

Consistent with the decrease in lipid droplets, expression of adipsin and adiponectin was down-regulated in the MafAsiRNA-treated cells. Adiponectin was recently reported to have insulin-sensitizing, anti-atherogenic and -inflammatory actions but no effect on insulin secretion (23). Adipsin is secreted by adipocytes, and adipsin release at the cell surface is stimulated by insulin. Adipsin is a transcriptional factor for adipocyte differentiation and contributes to host defense by functioning as factor $\mathrm{D}$ in the alternative pathway of complement activation (24). Adipsin and adiponectin are thought to cooperate in promoting adipocyte development (25). Moreover, although it is unclear how adipocytokines act at the local level, they are involved in metabolic mechanisms that allow cross talk with distant organs and the coordination of a variety of biological processes, including energy metabolism processes, the inflammatory cascade, and insulin-stimulated secretion. While the precise mechanisms and cross-links were not clarified in this study, Maf-regulated adipocytes appear to affect the adipokine network $(26,27)$.

Preliminary data from a microarray analysis revealed changes in the level of expression of several genes in MafAsiRNA-treated cells, with prominent down-regulated expression of the genes encoding liporotein lipase and latexin (28), suggesting possible roles of MafA in lipid metabolism and cell differentiation. We confirmed the down-regulation of lipoprotein lipase mRNA by real-time PCR (Fig. 6). Since we have not yet investigated the significance of related genes or systematically analyzed them, further investigation will be needed to understand MafA-related regulation of adipocytes.

In summary, suppression of MafA mRNA expression in adipocytes in vitro in this study prevented adipocyte differentiation and down-regulated several transcriptional factors and adipocytokines, and it altered the expression of genes related to lipid metabolism and cell differentiation.

\section{Acknowledgments}

The authors thank Keiko Shiratori, MD, for the scientific discussions, and Atsuko Teraoka for technical assistance. Parts of this work were presented in abstract form at the Meeting of the American Diabetes Association in June, 2008 in San Francisco, USA. This study was supported in part by a Grantin-Aid for Scientific Research (C;20591637, C;14571236) and by a Yayoi Yoshioka Award.

\section{References}

1. Nishizawa M, Kataoka K, Goto N, Fujiwara KT and Kawai S: v-maf, a viral oncogene that encodes a 'leucine zipper' motif. Proc Nat Acad Sci USA 86: 7711-7715, 1989.

2. Kawai S, Goto N, Kataoka K, Saegusa T, Shinno-Kohno H and Nishizawa $\mathrm{M}$ : Isolation of the avian transforming retrovirus, AS42, carrying the $\mathrm{v}$-maf oncogene and initial characterization of its gene product. Virology 188: 778-784, 1992.

3. Kataoka K, Nishizawa M and Kawai S: Structure-function analysis of the maf oncogene product, a member of the b-Zip protein family. J Virol 67: 2133-2141, 1993.

4. Olbrot M, Rud J, Moss LG and Sharma A: Identification of ß-cellspecific insulin gene transcription factor RIPE3b as mammalian MafA. Proc Natl Acad Sci USA 99: 6737-6742, 2002.

5. Kataoka K, Han S, Shioda S, Hirai M, Nishizawa M and Handa H: MafA is a glucose-regulated and pancreatic ß-cell-specific transcriptional activator for the insulin gene. J Biol Chem 277: 49903-49910, 2002.

6. Matsuoka T, Zhao L, Artner I, Jarrett HW, Friedman D, Means A and Stein R: Members of the large maf transcription family regulate insulin gene transcription in islet $\beta$ cells. Mol Cell Biol 23: 6049-6062, 2003.

7. Kataoka K, Shioda S, Ando K, Sakagami K, Handa H and Yasuda K: Differentially expressed maf family transcription factors, c-maf and mafA, activate glucagons and insulin gene expression in pancreatic $\alpha$ - and $\beta$-cells. J Mol Endocrinol 32: 9-20, 2004.

8. Matsuoka T, Artner I, Henderson E, Means A, Sander M and Stein R: The mafA transcription factor appears to be responsible for tissue-specific expression of insulin. Proc Natl Acad Sci USA 101: 2930-2933, 2004.

9. Zhao L, Guo M, Matsuoka T, Hagman DK, Parazzoli SD, Poitout V and Stein R: The islet $B$ cell-enriched mafA activator is a key regulator of insulin gene transcription. J Biol Chem 280: 11887-11894, 2005.

10. Harmon JS, Stein R and Robertoson RP: Oxidative stressmediated, post-translational loss of mafA protein as a contributing mechanism to loss of insulin gene expression in glucotoxic beta cells. J Biol Chem 280: 11107-11113, 2005.

11. Zhang C, Moriguchi M, Kajihara M, Esaki R, Harada Al, Shimohata H, Oishi H, Hamada M, Morito N, Hasegawa K, Kudo T, Engel JD, Yamamoto $M$ and Takahashi S: MafA is a key regulator of glucose-stimulated insulin secretion. Mol Cell Biol 25: 4969-4976, 2005.

12. Tsuchiya M, Taniguchi S, Yasuda K, Nitta K, Maeda A, Shigemoto $\mathrm{M}$ and Tsuchiya K: Potential roles of large mafs in cell lineages and developing pancreas. Pancreas 32: 408-416, 2006.

13. Sakai M, Imaki J, Yoshida K, Ogata A, Matsushima HY, Nishizawa $M$ and Nishi $S$ : Rat maf related genes: specific expression in chondrocytes, lens and spinal cord. Oncogene 14: 745-750, 1997.

14. Chesi M, Bergsagel PL, Shonukan OO, Martelli ML, Brents LA, Chen T, Schrock E, Ried T and Kuehl WM: Frequent dysregulation of the c-maf proto-oncogene at 16q23 by translocation to an Ig locus in multiple myeloma. Blood 91: 4457-4463, 1998. 
15. MacLean EH, Kim JI, Glimcher MJ, Wang J, Kronenberg HM and Glimcher LH: Absence of transcription factor c-maf causes abnormal terminal differentiation of hypertrophic chondrocytes during endochondral bone development. Dev Biol 262: 51-63, 2003.

16. Ronti T, Lupattelli G and Mannarino E: The endocrine function of adipose tissue: an update. Clin Endocrinol 64: 355-365, 2006.

17. Tsuchiya M, Yoshida T, Taniguchi S, Yasuda K, Maeda A, Hayashi A, Tanaka J, Shigemoto M, Nitta K and Tsuchiya K: In vivo suppression of mafA mRNA with siRNA and analysis of the resulting alteration of the gene expression profile in mouse pancreas by the microarray method. Biochem Biophys Res Commun 356: 129-135, 2007.

18. Yoshida T, Tang SS, Hsiano LL, Jensen RV, Ingelfinger JR and Gullans SR: Global analysis of gene expression in renal ischemia-reperfusion in the mouse. Biochem Biophys Res Commun 291: 787-794, 2002.

19. Farmer SR: Transcriptional control of adipocyte formation. Cell Metab 4: 263-273, 2006.

20. Serria MS, Ikeda H, Omoteyama K, Hirokawa J, Nishi S and Sakai M: Regulation and differential expression of the c-maf gene in differentiating cultured cells. Biochem Biophys Res Commun 310: 318-326, 2003.

21. McCaffrey AP, Meuse L, Pham TT, Conklin DS, Hannon GJ and Kay MA: RNA interference in adult mice. Nature 418: 38-39, 2002.
22. Kobayashi N, Matsui Y, Kawase A, Hirata K, Miyagishi M, Taira K, Nishikawa $M$ and Takakura Y: Vector-based in vivo RNA interference: dose- and time-dependent suppression of transgene expression. J Pharmacol Exp Ther 308: 688-693, 2004.

23. Staiger K, Stefan N, Staiger H, Brendel MD, Brandhorst D, Bretzel RG, Machicao F, Kellerer M, Stumvoll M, Fritsche A and Haring HU: Adiponectin is functionally active in human islets but does not affect insulin secretory function or B-cell lipoapoptosis. J Clin Endocrinol Metab 90: 6707-6713, 2005.

24. Miner JL: The adipocyte as an endocrine cell. J Anim Sci 82: 935-941, 2004

25. Kadowaki T, Yamauchi T, Kubota N, Hara K, Ueki K and Tobe K: Adiponectin and adiponectinreceptors in insulin resistance, diabetes, and the metabolic syndrome. J Clin Invest 116: 1784-1792, 2006.

26. Poitout V, Hagman D, Stein R, Artner I, Robertson RP, Harmon JS: Regualtion of the insulin gene by glucose and fatty acids. J Nutr 136: 873-876, 2006.

27. Okamoto Y, Kihara S, Funahashi T, Matsuzawa Y and Libby Y: Adiponectin: a key adipocytokine in metabolic syndrome. Clin Sci 110: 267-268, 2006.

28. De Haan G: Latexin is a newly discovered regulator of hematopoietic stem cells. Nat Genet 39: 141-142, 2007. 